\title{
Infracolic Approach to the Superior Mesenteric Vessels for a Large Pancreatic Tumor with Right Colon Invasion - A Case Report and Literature Review
}

\author{
NICOLAE BACALBASA ${ }^{1}$ and IRINA BALESCU ${ }^{2}$ \\ ${ }^{1}$ Department of Obstetrics and Gynecology, \\ Carol Davila University of Medicine and Pharmacy, Bucharest, Romania; \\ ${ }^{2}$ Department of General Surgery, Ponderas Academic Hospital, Bucharest, Romania
}

\begin{abstract}
Pancreatic cancer remains one of the most aggressive malignancies and is frequently diagnosed in advanced stages when local invasion is already present. In such cases, curative resection is often not possible; however, in certain cases, local invasion will not preclude radical surgery. We present the case of a 63-year-old patient who was diagnosed with a large pancreatic tumor with right colonic invasion in whom a pancreatoduodenectomy en bloc with right colectomy was successfully performed.
\end{abstract}

Pancreatic cancer remains one of the most aggressive malignancies, with a low rate of long-term survival. Pancreatic cancer has a peak incidence between 60 and 80 years of age and most often induces insidious symptoms such as non-specific epigastric pain, weight loss, nausea or asthenia $(1,2)$. In more advanced stages, specific symptoms such as jaundice or upper digestive stenosis might occur; however, in most of cases, local invasion into the surrounding vascular elements, such as hepatic pedicle, celiac axis or superior mesenteric vessels, occurs and the tumor becomes borderline resectable or even unresectable (3-6). For this reason, some authors recommend an infracolic approach in order to assess the tumor relationship with the mesenteric vessels; moreover, it seems that this approach is especially needed in cases presenting anterior invasion of the right and transverse colon.

Correspondence to: Nicolae Bacalbașa, Dimitrie Racoviță Street, no. 2, Bucharest, Romania. Tel: +40 723540426, e-mail: nicolae_ bacalbasa@yahoo.ro

Key Words: Pancreatic cancer, pancreatoduodenectomy, right colectomy.

\section{Case Report}

A 63-year-old patient presented for diffuse epigastric pain and weight loss; the preoperative upper digestive endoscopy revealed the presence of an extrinsic stenosis at the level of the descending part of the duodenum, while the lower digestive endoscopy revealed an extrinsic stenosis of the right colonic angle. Preoperative computed tomography showed the presence of a large tumor originating from the pancreatic head with duodenal and right colonic invasion; no sign of vascular invasion was described. The biochemical studies revealed a high level of cancer antigen 19-9 $(892 \mathrm{U} / \mathrm{ml})$, orientating the diagnosis to a locally advanced pancreatic tumor with right colon invasion. The patient was submitted to surgery; intraoperatively the presence of a large tumor of the pancreatic head with right colonic invasion was observed; in order to achieve complete resection of the tumoral mass with negative resection margins, an infracolic approach to the tumor was performed (Figures 1-5); the tumor was resected en bloc with cephalic pancreatoduodenectomy and right colectomy (Figure 6). The postoperative course was uneventful, the patient being discharged on the seventh postoperative day. The patient was submitted to six cycles of adjuvant chemotherapy; at one year follow-up, the patient is free of any recurrent disease.

\section{Discussion}

Pancreatoduodenectomy remains the treatment-of-choice for ampullary and pancreatic head adenocarcinomas; however this is a demanding procedure, with significant rates of postoperative complications even in high-volume centers $(7,8)$. Due to the improvement of surgical techniques, pancreatic resections have lately been performed with lower rates of morbidity (7-9); as a consequence, the rate of long-term survival has 
significantly increased and offers the surgeon the possibility of associating this procedure with other visceral resections without increasing the rate of complications (1012). Moreover, local extension of the tumor into the surrounding viscera is no longer an absolute contraindication, being more related to the initial location of the tumor than to the biological aggressiveness of the malignant process $(5,6,12,13)$. Pancreatoduodenectomy has recently been associated with other visceral resections in order to obtain an R0 resection in both locally advanced and metastatic disease, with acceptable rates of postoperative complications, demonstrating in this way that although it is a demanding procedure, pancreatoduodenectomy can be safely performed and can significantly improve survival whenever complete resection is achievable $(14,15)$.

From the anatomical point of view, the pancreatic head is situated in close proximity to the great abdominal vessels (abdominal aorta and inferior vena cava) posteriorly, superior mesenteric vessels medially and transverse mesocolic root inferiorly (15). Tumors located on the anterior surface of the pancreatic head are more likely to invade the right colic flexure or the transverse colon and mesocolic surface; this specific site of invasion can be revealed preoperatively during imaging studies and confirmed intraoperatively, after lifting the greater omentum cephalad and assessing the duodeno-jejunal angle. Whenever invasion of this area is present but an R0 resection is achievable, some authors recommend performing an en bloc resection of the pancreatic head and of the invaded area of the colon (16). In these cases, an infracolic approach to the superior mesenteric vessels is recommended, followed by an en bloc mobilization of the transverse colon and pancreatic head. In the study conducted by Alvarado et al. and published in 2010, the authors reviewed 156 cases of patients submitted to pancreatoduodenectomy, nine of them necessitating association of en bloc colic resection. An R0 resection was achieved in all cases, while the histopathological studies revealed the presence of positive lymph nodes in three out of the nine cases; regarding the rate of postoperative complications, it was similar with that reported for the pancreatoduodenectomy group. As for the long-term outcomes, the authors reported a median survival rate of 26.5 months, a single patient being diagnosed with recurrent disease (15).

In another similar study conducted by Eric Kimchi et al. and published in 2009, the data of 180 patients submitted to pancreatoduodenectomy were reviewed, revealing 14 cases in which an en bloc right colectomy in association with pancreatoduodenectomy was performed. The peculiarity of that study was the fact that these extended visceral resections were not imposed in all cases by the presence of a pancreatic

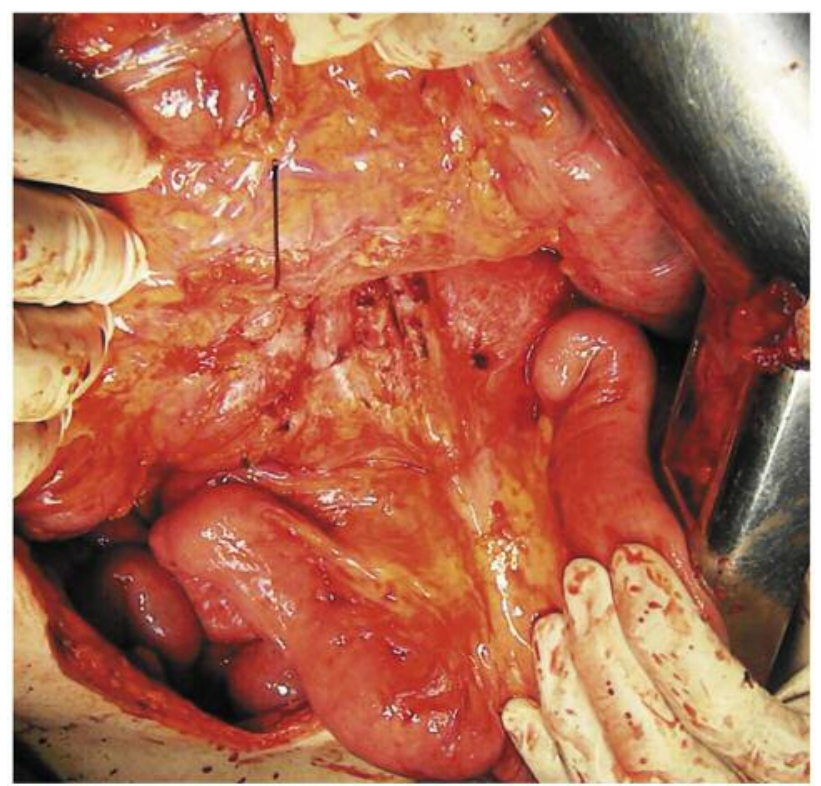

Figure 1. Initial intraoperative aspect - infracolic approach to the superior mesenteric vessels in a patient with pancreatic head tumor with right colonic invasion.

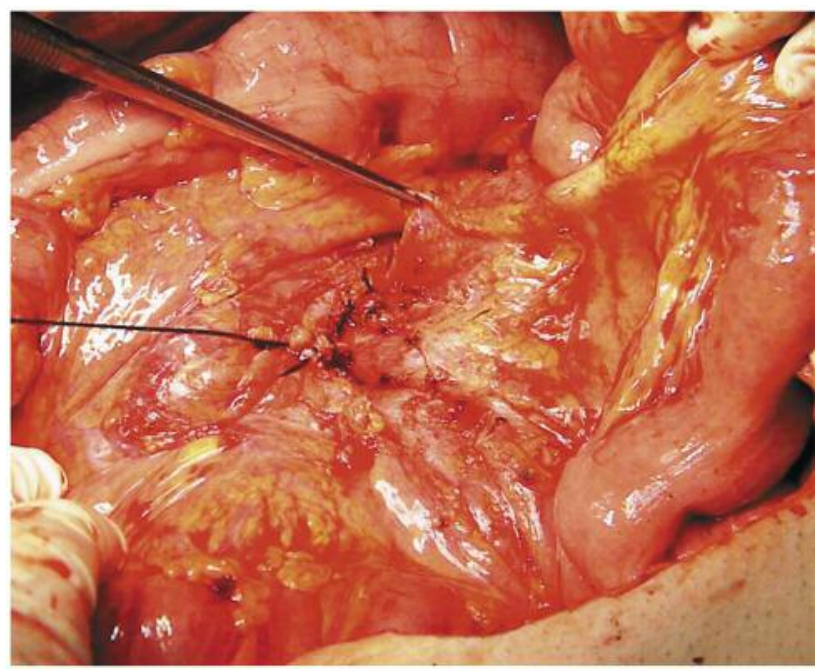

Figure 2. Dissecting the superior mesenteric vessels.

tumor; pancreatic adenocarcinoma was found in eight out of the 14 cases, while the other patients were diagnosed with retroperitoneal sarcomas (in two cases) locally invasive colonic cancer (in two cases), duodenal gastrointestinal stromal tumor (in one case) or duodenal adenocarcinoma (in one case). Negative resection margins were achieved in 11 out of the 14 cases. Regarding the postoperative outcomes, eight 


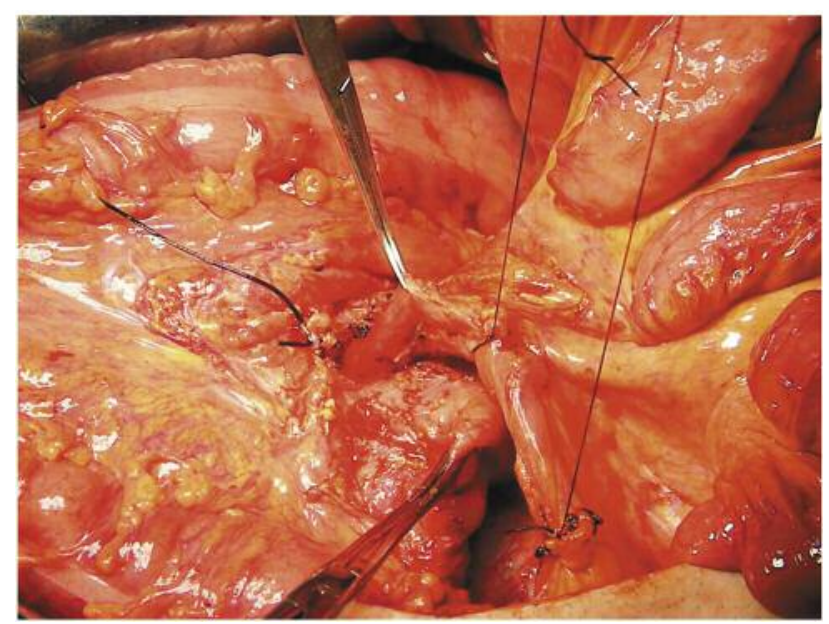

Figure 3. Mobilizing the tumor en bloc with pancreatoduodenectomy and right colectomy.

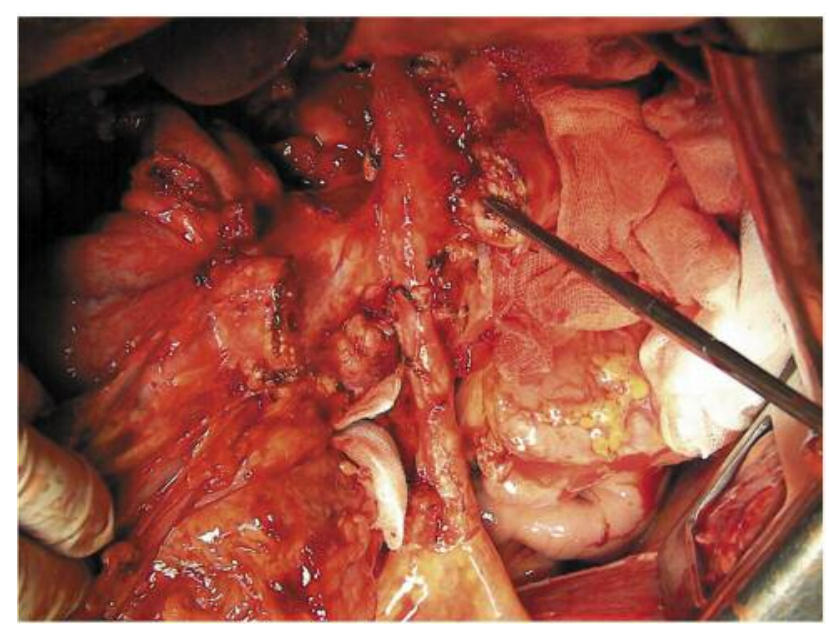

Figure 4. The final aspect after dissection of the superior mesenteric vessels and pancreatic transection.

out of the 14 cases were diagnosed with postoperative complications (ranging from delayed gastric emptying to pancreatic fistula), while the mean hospital stay was 8 days; considering the long-term outcomes of the patients diagnosed with pancreatic tumors, the median survival was 20 months (12). The authors concluded that association of right hemicolectomy with pancreatoduodenectomy in order to achieve an R0 resection for locally advanced pancreatic cancer can be safely performed, with good long-term outcomes and similar median survival rates to those reported for patients submitted to standard pancreato-duodenectomy; moreover, the

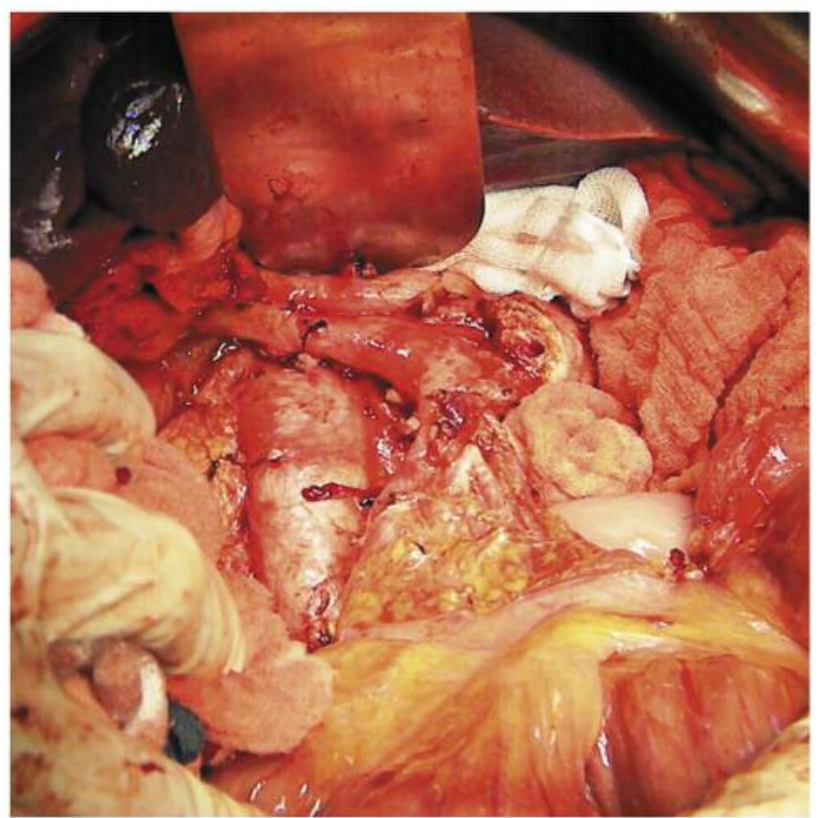

Figure 5. The final aspect after pancreatoduodenectomy en bloc with right colectomy and lymph node dissection.

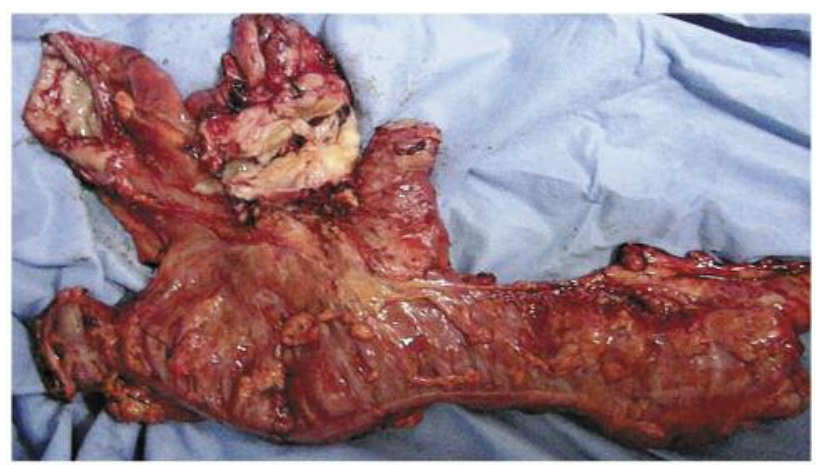

Figure 6. The specimen from pancreatoduodenectomy en bloc with right colectomy.

outcome of these patients is significantly better when compared to those for which surgery is not performed $(8,17$, 18). The authors also considered that the high percentage of cases submitted to pancreato-duodenectomy en bloc with right colectomy reported in their studies was rather related to the authors' interest in achieving a complete resection in locally advanced pancreatic cancer. The low rate of severe postoperative complications, as well as the median length of hospital stay of 8 days, suggests that right colectomy can be safely associated with pancreatoduodenectomy in order to maximize surgical effort (12). 
The infracolic approach to large pancreatic tumors seems to be a good choice of surgical technique for evaluating the resectability of locally advanced pancreatic tumors even in the absence of colic invasion. Described for the first time by Weitz et al., the infracolic approach allows safe and clear identification of the superior mesenteric artery and of the superior mesenteric vein from the infracolic position; afterwards the dissection is continued cranially. According to the same author, this approach is especially useful in cases with locally advanced pancreatic tumors with suspected tumoral infiltration of the superior mesenteric artery (19).

Referred to by Zhu et al. under the generic name of "inferior infracolic 'superior mesenteric artery first' approach with a notouch isolation", the method is very efficient in predicting the possibility of an R0 resection. According to these authors, this method was used in 21 cases with borderline resectable lesions between 2014 and 2015. In five cases, the invasion of the superior mesenteric artery imposed ending the procedure; in 14 cases, a pancreatoduodenectomy was successfully performed, while in another two cases, the presence of positive resection margin on the pancreatic neck necessitated performing a total pancreatectomy. Moreover, venous resection was needed in eight cases, while an $\mathrm{R} 0$ resection was achieved in all the cases included in the study. The authors reported a 1-year survival rate of $75 \%$, demonstrating the capability of this technique to predict the possibility of achieving an R0 resection in patients with borderline resectable pancreatic head tumors (20).

\section{Conclusion}

The infracolic approach for locally invasive pancreatic tumors represents a safe surgical technique for large pancreatic tumors with right colonic invasion, providing easy access to the superior mesenteric vessels. Once the absence of tumoral arterial infiltration is demonstrated, the tumor can be safely removed by performing a pancreatoduodenectomy en bloc with right colectomy. Moreover, studies reported so far demonstrate that if an $\mathrm{R} 0$ resection is achieved with the association of colonic resection, a significant survival benefit is expected, while the rate of postoperative complications remains similar to those reported for cases submitted to standard pancreatoduodenectomy.

\section{Acknowledgements}

This work was supported by a grant of the Romanian National Authority for Scientific Research and Innovation, CNCS UEFISCDI, project number PN-II-RU-TE-2014-4-2533.

\section{References}

1 Ansari D, Tingstedt B, Andersson B, Holmquist F, Sturesson C, Williamsson C, Sasor A, Borg D, Bauden M and Andersson R: Pancreatic cancer: yesterday, today and tomorrow. Future Oncol 12: 1929-1946, 2016.
2 Mirancea GV, Morosanu AM, Carniciu S, Dima S, Bacalbasa N, Popescu I, Ionescu-Tirgoviste C and Mirancea N: Relevant infrastructural alterations in a pancreatic neuroendocrine tumor: an insulinoma case. Rom J Morphol Embryol 55: 659$668,2014$.

3 National Comprehensive Cancer Network. Clinical Practice Guidelines in Oncology. Pancreatic Adenocarcinoma. Version 2 (2015).

4 Bockhorn M, Uzunoglu FG, Adham M, Imrie C, Milicevic M, Sandberg AA, Asbun HJ, Bassi C, Buchler M, Charnley RM, Conlon K, Cruz LF, Dervenis C, Fingerhutt A, Friess H, Gouma DJ, Hartwig W, Lillemoe KD, Montorsi M, Neoptolemos JP, Shrikhande SV, Takaori K, Traverso W, Vashist YK, Vollmer C, Yeo CJ and Izbicki JR: Borderline resectable pancreatic cancer: a consensus statement by the International Study Group of Pancreatic Surgery (ISGPS). Surgery 155: 977-988, 2014.

5 Brasoveanu V, Anghel C, Barbu I, Pautov M, Ionescu MI, Motthor M, Balescu I, Dima $\mathrm{S}$ and Bacalbasa N: Pancreatoduodenectomy en bloc with portal and superior mesenteric artery resection-a case report and literature review. Anticancer Res 35: 1613-1618, 2015.

6 Brasoveanu V, Dumitrascu T, Bacalbasa N and Zamfir R: Splenic artery used for replaced common hepatic artery reconstruction during pancreatoduodenectomy - a case report. Chirurgia 104: 499-504, 2009.

7 Sohn TA, Yeo CJ, Cameron JL, Koniaris L, Kaushal S, Abrams RA, Sauter PK, Coleman J, Hruban RH and Lillemoe KD: Resected adenocarcinoma of the pancreas-616 patients: results, outcomes, and prognostic indicators. J Gastrointest Surg 4: 567579, 2000.

8 Cameron JL, Riall TS, Coleman J and Belcher KA: One thousand consecutive pancreaticoduodenectomies. Ann Surg 244: 10-15, 2006.

9 Bacalbasa N, Dima S, Balescu I, David L, Brasoveanu V and Popescu I: Results of primary cytoreductive surgery in advanced-stage epithelial ovarian cancer: a single-center experience. Anticancer Res 35: 4099-4104, 2015.

10 Suzuki Y, Fujino Y, Tanioka Y, Sakai T, Ajiki T, Ueda T, Tominaga $\mathrm{M}$ and Kuroda $\mathrm{Y}$ : Resection of the colon simultaneously with pancreaticoduodenectomy for tumors of the pancreas and periampullary region: short-term and long-term results. World J Surg 28: 1007-1010, 2004.

11 Saiura A, Yamamoto J, Ueno M, Koga R, Seki M and Kokudo $\mathrm{N}$ : Long-term survival in patients with locally advanced colon cancer after en bloc pancreaticoduodenectomy and colectomy. Dis Colon Rectum 51: 1548-1551, 2008.

12 Kimchi ET, Nikfarjam M, Gusani NJ, Avella DM and StaveleyO'Carroll KF: Combined pancreaticoduodenectomy and extended right hemicolectomy: outcomes and indications. HPB 11: 559-564, 2009.

13 Botezatu A, Bleotu C, Nastase A, Anton G, Bacalbasa N, Duda D, Dima SO and Popescu I: Epigenetic silencing of GNMT gene in pancreatic adenocarcinoma. Cancer Genomics Proteomics 12: 21-30, 2015.

14 Bacalbasa N, Balescu I, Dima S, Brasoveanu V and Popescu I: Pancreatic resection as part of cytoreductive surgery in advanced-stage and recurrent epithelial ovarian cancer-a single-center experience. Anticancer Res 35: 4125-4129, 2015 . 
15 Alvarado-Bachmann R, Choi J, Gananadha S, Hugh TJ and Samra JS: The infracolic approach to pancreatoduodenectomy for large pancreatic head tumours invading the colon. Eur J Surg Oncol 36: 1220-1224, 2010.

16 Howard TJ, Krug JE, Yu J, Zyromski NJ, Schmidt CM, Jacobson LE, Madura JA, Wiebke EA and Lillemoe KD: A margin-negative R0 resection accomplished with minimal postoperative complications is the surgeon's contribution to long-term survival in pancreatic cancer. J Gastrointest Surg 10: 1338-1345, 2006

17 Yekebas EF, Bogoevski D, Cataldegirmen G, Kunze C, Marx A, Vashist YK, Schurr PG, Liebl L, Thieltges S, Gawad KA, Schneider C and Izbicki JR: En bloc vascular resection for locally advanced pancreatic malignancies infiltrating major blood vessels: perioperative outcome and long-term survival in 136 patients. Ann Surg 247: 300-309, 2008.
18 Kuhlmann KF, De Castro SM and Gouma DJ: Surgical palliation in pancreatic cancer. Minerva Chir 59: 137-149, 2004.

19 Weitz J, Rahbari N, Koch M and Buchler MW: The 'artery first' approach for resection of pancreatic head cancer. J Am Coll Surg 210: e1-e4, 2010.

20 Zhu J, Han D, Li X, Pan F, Ma J, Kou J, Fan H, Lang R and He Q: Inferior infracolic 'superior mesenteric artery first' approach with a no-touch isolation surgical technique in patients with a borderline resectable cancer of the pancreatic head. Ann Surg Oncol 23: 976-980, 2016 